\title{
Preoperative localization of solitary pulmonary nodule: a narrative review
}

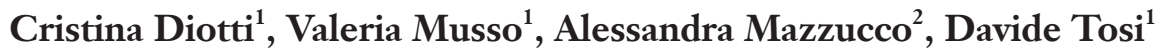 \\ ${ }^{1}$ Thoracic Surgery and Lung Transplantation Unit, Fondazione IRCCS Ca' Granda Ospedale Maggiore Policlinico, University of Milan, Milan, \\ Italy; ${ }^{2}$ Thoracic Surgery Unit, University of Milan-ASST Santi Paolo e Carlo, Milan, Italy \\ Contributions: (I) Conception and design: D Tosi; (II) Administrative support: All authors; (III) Provision of study materials or patients: All authors; \\ (IV) Collection and assembly of data: All authors; (V) Data analysis and interpretation: All authors; (VI) Manuscript writing: All authors; (VII) Final \\ approval of manuscript: All authors. \\ Correspondence to: Davide Tosi, MD. Thoracic Surgery and Lung Transplantation Unit, Fondazione IRCCS Ca' Granda Ospedale Maggiore \\ Policlinico, 20122 Milan, Italy. Email: davide.tosi@policlinico.mi.it.
}

\begin{abstract}
The diffusion of lung cancer screening programs using computed tomography (CT) scan and the improvement in imaging technologies have led to an increased number of indeterminate solid and subsolid small lung lesions, in which percutaneous or bronchoscopic diagnostic procedures may be unsuccessful or not technically feasible. In these cases, a surgical resection using minimally invasive techniques can be indicated, with both diagnostic and therapeutic purposes. Intraoperative detection of small lung nodules is still a challenge for surgeons, especially in the era of video and robot-assisted thoracic surgery, which could make direct finger palpation of lung parenchyma through thoracoscopic incisions demanding and thus require conversion to a standard thoracotomy to find the lesion. In this scenario, several preoperative and intraoperative localization techniques for small lung nodules are available, from image-guided techniques with percutaneous markers placement or injection of liquid agents (dyes, radio-tracers), to novel promising marking methods using electromagnetic navigation bronchoscopy (ENB) or Hybrid Room technology. The proper localization technique should be selected according to the lesion's position, size, and radiological characteristics, as well as the expertise and the preferences of the staff performing preoperative and surgical procedures. The aim of this narrative review is to describe and compare various available preoperative and intraoperative localization techniques in terms of safety, possible complications, cost/effectiveness and feasibility in everyday clinical practice, and to provide information on this topic for clinicians dealing with non-palpable or deep-located small lung nodules.
\end{abstract}

Keywords: Non-palpable lung nodule; solitary lung nodule; preoperative localization techniques; video-assisted thoracic surgery (VATS)

Received: 20 April 2021; Accepted: 02 June 2021; Published: 30 December 2021.

doi: 10.21037/asj-21-17

View this article at: https://dx.doi.org/10.21037/asj-21-17

\section{Introduction}

In the last three decades, there has been an exponential increase in the finding of small pulmonary nodules, thanks to the diffusion of screening programs and advances in computed tomography (CT) technology. Diagnosis and treatment of these indeterminate nodules can be challenging: malignancy risk score models (1) and deep learning software (2) are used in assessing malignancies, and often lead to invasive diagnostics. A definitive diagnosis can be difficult to obtain using conventional bronchoscopy or fine needle ago biopsy (FNAB) (3). Moreover, in the era of personalized medicine, a large amount of tissue may be needed to precisely define the tumour's mutational pattern. In these cases, a wedge resection via video-assisted thoracic surgery (VATS) can be indicated, as a diagnostic intervention, or as a first step preceding an anatomical 
resection.

Target nodules can be difficult to palpate through a small incision, especially if they are ground grass opacity (GGO), semisolid, or located deep in the parenchyma; in these cases, conversion to thoracotomy can be necessary. In order to avoid this occurrence, various techniques have been proposed to identify the nodule before or during VATS procedure: preoperative CT-guided placement of localizers, intraparenchymal injection of liquid agents, electromagnetic navigation bronchoscopy (ENB) and intraoperative ultrasound (US).

A recent expert consensus workshop report on guidelines for preoperative assisted localization of small pulmonary nodules recommended the following indications:

(I) Solitary or multiple small (i.e., diameter $<15 \mathrm{~mm}$ ) or deep (i.e., $>10 \mathrm{~mm}$ from visceral pleura) pulmonary nodules;

(II) Pure ground-glass nodule or subsolid nodule;

(III) Difficult to localize nodules via intraoperative palpation (4).

The purpose of this article is to describe the current clinical practice in preoperative localization of solitary pulmonary nodule (SPN) and to compare the techniques in terms of feasibility, safety and complications. We present the following article in accordance with the Narrative Review reporting checklist (available at https://asj.amegroups.com/ article/view/10.21037/asj-21-17/rc).

\section{Methods}

A literature search was conducted by the authors to identify all English-written published articles on localization of lung lesions. PubMed, EMBASE and Web of Science databases were consulted matching the terms "lung nodule" and "localization" and "non-palpable" with "AND" until 31th January 2021. The search was extended by consulting the listed references of each article.

All the articles, case reports and case series were included in this narrative review. Abstracts were excluded. The extracted data included study characteristics, number of patients, clinical data, type of procedure, results and complications.

\section{Preoperative and intraoperative localization techniques}

\section{Computed tomography percutaneous marker placement}

Various fiducial markers can be used, and placed in the lung parenchyma under CT guidance. This technique is generally safe and affordable, and is usually performed by surgeons or trained radiologists.

Hook wire guided localization technique was first used for breast nodules, and then applied to lung lesions too (5). A first CT scan is obtained in order to identify the nodule and the most suitable position for the patient during the procedure. The device consists of a circular flexible hook wire connected to a long suture thread; the hook is inserted in a 20 Gauge needle, and it takes back its circular configuration once it has been deployed, therefore anchoring into the nodule. Hook wires usually have a pre-shaped form, with one or two thorns, while spiral wires have a circular configuration. The correct puncture site is determined and, after administration of local anaesthetic, the device is gradually advanced into the lung nodule under CT guidance. Once the correct location of the marker is confirmed by CT, the hook wire is deployed and the needle is withdrawn. CT imaging is employed to rule out immediate complications, such as pneumothorax and haemothorax. The thread connected to the wire, which remains outside of the patient, is covered with sterile gauze and left unsecured so that it is allowed to follow the lung's movement and to avoid the hook's dislodgement. The patient is then moved to the operating room. During VATS resection, the hook is identified under direct vision. Hanauer et al. retrospectively review their experience with the hook wire technique on 181 patients and 187 nodule resections. They reported a $3.7 \%$ of hook wire dislodgement: nonetheless, the nodules were identified by locating the lung puncture site and resected. Pneumothorax occurred in $35.9 \%$ of cases: in 4 patients, all of whom had emphysema, this complication required a small chest tube placement. Lung tissue bleeding, with no clinical impact, was observed in $5.9 \%$ (6). Patella et al. reported the results of a single centre experience on 93 patients (102 cases) employing spiral wires for lung nodule localization: the prevalence of pneumothorax was $4.9 \%$, while haemorrhage occurred in $18.6 \%$. Dislodgement rate was $2.9 \%$. The authors claimed that spiral wires have better performances than hook wires (7).

Microcoils implantation is a widely used technique to preoperatively mark lung nodules. A CT scan is obtained to locate the nodule and plan the access route during the procedure. The acquired CT scan also provides information regarding the distance between the nodule and the nearest pleural surface, the exact lobar and segmental location, possible closeness to vascular structures, and the presence of emphysema. Based on the first CT scan, the patient is placed on the CT table in the correct position in order to 
facilitate needle insertion. A fiber-coated platinum microcoil is loaded into a 20 -Gauge $10-15 \mathrm{~mm}$ long graduated coaxial introducer. After local anaesthesia, the loaded coaxial is inserted under CT guidance and pushed through or in proximity of the lung nodule. The distal portion of the microcoil is deployed and, after ensuring proper placement through CT imaging, the introducer is withdrawn and the entire microcoil is released into the parenchyma. Before transferring the patient to the operating room, another CT is performed to confirm the correct position of the microcoil into the nodule and to investigate the presence of possible procedure-related complications such as haemorrhage and pneumothorax. During VATS surgery, the fiducial marker's position is identified using fluoroscopy, and the stapler is placed deep to the microcoil. An X-ray of the specimen can be of aid to confirm the complete removal of the microcoil. McGuire et al. reported a 5-year update following the end of their randomized controlled trial on microcoil-guided lung resection: they successfully identified all 97 nodules in 92 patients. Their study described a quite high frequency of complications, none of which required treatment: pneumothorax occurred in $46.4 \%$ of patients, lung haematoma in $18.6 \%$, microcoil dislodgement in $4.1 \%$, and haemoptysis in $2.1 \%$ of cases (8).

An hydrogel plug, initially designed to prevent pneumothorax during percutaneous lung biopsies, has recently been employed as a fiducial marker for preoperative lung nodules localization. The delivery system is set at the correct skin-to-pleural distance after measuring on CT scan; through a 17 Gauge or 19 Gauge coaxial, the dry plug is then deployed into the lung nodule using the delivery system. Due to hydration by contact with the lung tissue, the plug expands and fills the needle tract, thus closing the pleural puncture and reducing the risk of pneumothorax after the procedure. A portion of the plug protrudes from the pleural surface and marks the nodule site. Imperatori et al. published their experience with this technique on 27 patients with 28 pulmonary nodules. In 3 cases (11\%), a plug displacement occurred, but the nodule was nonetheless located and resected through palpation or identification of the pleural puncture hole. Notably, in 2 out of these 3 cases, surgery was performed 7 and 10 days after the percutaneous CT-guided plug placement. Pneumothorax requiring chest tube placing occurred in $4 \%$ of cases (9).

\section{Percutaneous injection of different types of liquid agents}

A widely used technique for preoperative localization is the CT-guided percutaneous injection of liquid substances, such as methylene blue, lipiodol, and radio-tracer. A CT scan is performed to identify the nodule, and under local anaesthesia, place the needle in the correct position. Then, the liquid substance is injected near the nodule and preferably also on the pleural surface, in such a way as to be visible during the surgery. At the end of the procedure, a CT scan is repeated to rule out possible complications, such as pneumo- or hemothorax. The identification of the nodule during surgery can be done by direct visualization (methylene blue), or by using either a C-arm fluoroscope (lipiodol) or an intraoperative probe (Tc $99 \mathrm{~m}$ radiotracer or iodine seed). Each technique has its own difficulties related to the time that elapses between the marking and the surgery. Indeed, some of these markers are especially limited by the possibility for them to spread widely away from the nodule: the time between marking and surgery must therefore be as short as possible. Furthermore, some studies revealed how difficult it is to identify the pulmonary nodule after labelling it with methylene blue. This is due to the rapid dispersion of the substance, making it necessary to perform the surgery in the shortest possible time following the marking (10). Conversely, since lipiodol is an oil-based iodinated contrast medium, it can remain identifiable even for weeks. Thus, the operation can be performed even days later, and the nodule is identified as a radiopaque region using fluoroscopy (11). Other surgeons utilize radionuclides, such as Tc $99 \mathrm{~m}$ radiotracer (12) or iodine seed (I-125) (13). Then, an intraoperative probe detects gamma ray emissions, and the nodule can be resected. Once the lesion is removed, the radio probe is used to confirm the absence of a strong signal in the remaining lung and also to determine the site of maximum activity in the specimen. However, the execution of the procedure depends on the presence of nuclear medicine equipment and staff in the operating room.

\section{ENB}

ENB is a relatively new navigation method: it enables the localization and marking of peripheral pulmonary lesions through dye or contrast medium injection, or by placing a fiducial marker. A preoperative CT scan is obtained and analysed by a dedicated software which creates a $3 \mathrm{D}$ airway tree, thus allowing to plan a navigation route to the nodule. The procedure is performed under general anaesthesia, using a single-lumen endotracheal tube for mechanical ventilation; the patient is placed in supine position on the 
electromagnetic board. The bronchoscope is placed into the correct segmental bronchus; the locatable electromagnetic guide and the extended working catheter are then advanced distally into the airways following the $3 \mathrm{D}$ real-time navigational pathway shown on the console screen. During the procedure, the distance between the guide and the nodule is measured. Once the target lesion has been located, the electromagnetic guide is removed, leaving the working catheter in place. The dye or fluorophore, depending on the preferred technique, is then injected through the catheter and into the lung close to the nodule. Moreover, ENB can be used to guide fiducial marker placement. After localization and marking, the anaesthesiologist replaces the tube with a double-lumen endotracheal tube, the patient is placed in lateral decubitus position, and nodule resection by VATS is performed. In a propensity-matched study by Kuo et al., the authors compared 15 and 30 patients undergoing ENB and CT-guided dye marking respectively. No statistically significant differences were found between the two groups in terms of localization time, surgery time, and complications. Failure to locate the nodule marking occurred in $6.7 \%$ cases in the ENB group, and in $10 \%$ of cases in the CT group. This data confirm the safety and feasibility of this technique (14).

\section{Hybrid room technology}

A hybrid operating room (HOR) is a high-technology space, which integrates Cone-beam CT (CBCT) and the operating room, allowing the carrying out of radiological and surgical manoeuvres in the same place. It is capable of providing real-time patient images, facilitating both the localization of non-palpable lung nodules and resection procedures with a minimally invasive surgery. The advantage is to perform these two procedures in the same room, thus reducing the complications rate, such as pneumothorax, metallic marker displacement, and dye diffusion. As a result, surgeons could perform excisions precisely and safely, even for multifocal lesions, and spare pulmonary tissue. This procedure is called image-guided video-assisted thoracoscopic surgery (iVATS) (15). The novelty of iVATS lies in the possibility of carrying out the localization of the nodule in real time, in the same suite, and then immediately proceed with the resection in a HOR. The anesthetized patient, with a double lumen tube in place, is placed in lateral decubitus and prepared for a VATS procedure. Performing the localization under general anaesthesia, especially in the lateral decubitus position, has the advantage of reducing patient's discomfort and shortening the time from marker insertion to the start of the VATS procedure. It is important to position the patient correctly, in order to avoid collisions with the rotating $\mathrm{C}$-arm of robotic angiography system that will acquire the CT images. The collaboration between radiologists and surgeons is essential to confirm the presence of the lesion and to plan the trajectory for the percutaneous positioning of the marker. The trajectory is conducted perpendicular to the nearest pleural surface avoiding anatomical obstacles, such as the ribs and scapula. Target position and intraoperative strategy determine marker selection (hookwire or coil). At this point, the surgical time can begin: mono-pulmonary ventilation is started before pleural incision. The localization of the marker is confirmed by intraoperative CBCT scan or fluoroscopy. It is also used to confirm that the marker is inside the specimen and to cross-examine the relative position between the marker and stapler before firing $(16,17)$.

\section{Intraoperative ultrasonography (US)}

US is a safe technique providing real-time imaging without ionizing radiation exposure. The idea of adopting intraoperative US evaluation during thoracoscopic surgery stemmed from its prior employment in laparoscopy. At first, there were some concerns regarding US of the pulmonary tissue, as the air in the lungs can hamper the diagnostic accuracy and cause artifacts, but this obstacle was overcome by the use of high-frequency ultrasound probes and by completely deflating the lung during US. For the same reason, nodule localization via US can be demanding in patients with pulmonary emphysema. This technique is mostly used to locate small solid pulmonary nodules, which can be determined by the presence of hyperechogenic lesions with acoustic shadowing. Nonetheless, some studies report US to be effective for the intraoperative identification of ground glass opacities and the evaluation of resection margins too (18). A $10-\mathrm{mm}$ linear 5 to $10 \mathrm{MHz}$ linear probe with a flexible angulating tip is often used during VATS procedures. The probe's range of motion is designed to allow for the identification of posterior or basal nodules and for the studying of the fissures. In a prospective clinical trial assessing the use of US in patients undergoing VATS resection for non-subpleural pulmonary nodules, Khereba et al. report a $93 \%$ sensitivity and a $100 \%$ positive predictive value. In 20 out of 46 cases, direct visual thoracoscopic inspection, finger palpation, and instrument sliding technique failed to identify the nodules, 
Table 1 Main advantages and disadvantages of preoperative and intraoperative non-palpable lung nodules localization techniques

\begin{tabular}{|c|c|c|}
\hline Method & Advantages & Disadvantages \\
\hline $\begin{array}{l}\text { Radioguided techniques } \\
\left({ }^{99 \mathrm{~m}} \mathrm{Tc},{ }^{125} \mathrm{I} \text {-iodine seed) }\right.\end{array}$ & $\begin{array}{l}\text { High success rate ( }-100 \%) \text {; longer waiting time } \\
\text { between marking and surgery (up to } 24 \text { hours); } \\
\text { minimal operator-dependence }\end{array}$ & $\begin{array}{l}\text { Two-stage procedure; radiation exposure; nuclear } \\
\text { medicine equipment }\end{array}$ \\
\hline $\begin{array}{l}\text { Electromagnetic navigation } \\
\text { bronchoscopy (ENB) }\end{array}$ & $\begin{array}{l}\text { One-stage procedure; useful also for deeper nodules; } \\
\text { lower risk of pneumothorax (useful for bilateral lesions) }\end{array}$ & $\begin{array}{l}\text { Low cost-effectiveness; increased time under } \\
\text { general anaesthesia; highly trained operators }\end{array}$ \\
\hline Intraoperative ultrasound & $\begin{array}{l}\text { No radiation exposure; real-time imaging; } \\
\text { intraoperative procedure }\end{array}$ & $\begin{array}{l}\text { Operator-dependent; not useful for GGO; highly } \\
\text { trained operators }\end{array}$ \\
\hline
\end{tabular}

CT, computed tomography; GGO, ground grass opacity.

and US was the only successful method, thus preventing conversion to thoracotomy (19). Moreover, compared to CT-guided fiducial markers, US does not entail the risk of pneumothorax or bleeding. On the other hand, lung US requires training and experience, as this technique is operator-dependent.

\section{Discussion}

The increasing use of low-dose CT scan as a screening method to identify early-stage lung cancer has led to a relevant increase in small lung lesions, both solid and subsolid/ground-glass opacities. In case of small, subsolid or deep-located lung nodules suspicious for malignancy, when a preoperative diagnosis is difficult to obtain, a surgical resection with minimally invasive techniques (VATS, RATS) can be indicated. Intraoperative thoracoscopic identification of non-palpable lung nodules is still considered a surgical challenge, and thoracotomy remains the gold standard to examine lung parenchyma by direct finger palpation (20); however, nowadays several preoperative marking strategies are available to allow for the identification of non-palpable lesions during VATS.

The proper marking technique should be selected according to the lesion's position, size, and density: transthoracic CT-guided strategies can be applied in case of peripheral lung nodules, while bronchoscopic localization procedures (e.g., ENB-guided techniques) can also be used in case of central lesions. Intraoperative US-guided marking methods are usually employed to detect small or deeplocated lung lesions, but should be avoided in case of GGO lesions, because their density is similar to that of normal pulmonary tissue, even if collapsed during the surgery. Table 1 summarizes main advantages and disadvantages of different reported techniques.

The aim of this review is to describe and compare various available preoperative localization techniques in terms of safety, possible complications, cost/effectiveness and feasibility in everyday clinical practice.

Percutaneous CT-guided marking techniques are the most common, feasible, and less expensive preoperative localization strategies to identify peripheral non-palpable lung nodules (21). The marking procedure can be performed under local anaesthesia and is generally well tolerated.

Various materials may be used to tag the lesion, from dyes (methylene blue, India ink) and radio-opaque liquids (barium, lipiodol) to small solid devices (mainly hookwires and microcoils) that can be detected radioscopically with intraoperative fluoroscopy, by direct visualization, or by palpation.

The main advantages of CT-guided techniques are the 
availability in almost every multi-specialty medical centre, the high cost-effectiveness and the limited learning curve. The most evident disadvantages are the need to perform a two-stage procedure, composed by a marking time in the CT-suite and a surgical time in the OR. Since some complications could occur during this waiting time, a well-established workflow is required to optimize times between the two stages. The most frequently described complications of these procedures are pneumothorax (reported percentages range from $4 \%$ to $49 \%$ of cases, $1.2-6 \%$ requiring intervention and chest tube placement), pulmonary perilesional haemorrhage $(7-29.8 \%)$ and haemothorax (22): in particular, hookwire localization is associated with the highest complication rates, while microcoils are associated with lower rates of pneumothorax and haemorrhage, probably because of the thrombogenic effect due to the composition of their fiber coating (23). Another concern about CT-guided techniques is the radiation exposure during the localization procedure and the intraoperative identification of radio-opaque markers, both for patients and involved personnel: the radiation doses should be considered in the choice of the proper localization procedure. Considering hookwire technique, one of the main limits leading to failure of marking is wire dislodgement due to patients' movements between the localization procedure and the surgical resection, (ranging from $4.5 \%$ to $9 \%$ of cases) even if the hookwire is well anchored to the skin with Band-Aids (24). However, in some cases, the lesion can still be identified following the pleural hematoma on the surface of the lung. A rare but severe complication caused by fiducial markers' placement is vascular embolization through a thin pulmonary vein to the left ventricle (24): for this reason, it is important to avoid vascular structures of any calibre during the placement of markers. Regarding localization with dyes, care must be taken to detect possible allergic reactions, ranging from cutaneous rash to anaphylaxis, described using methylene blue; moreover, methylene blue is associated with some cases of pleuritic chest pain, due to the irritation of the pleura. Several studies comparing various CT-guided localization techniques in terms of success rate, complications, pain score, and procedure time were published. In particular, a retrospective review proposed by Hwang et al. comparing 45 hookwire vs. 54 microcoilmarked lung lesions showed a surgical success rate of $100 \%$ in both groups, but with significant shorter VATS surgical time $(\mathrm{P}=0.004)$, less reported chest pain $(\mathrm{P}<0.001)$ and a significantly smaller volume of surgical specimens $(\mathrm{P}=0.043)$ in microcoil group, with no significant difference in CTguided procedure time (16.7 vs. 16.4 minutes; $\mathrm{P}=0.74$ ). Dislodgement occurred in 4 patients, all in the hookwire group; no major complications were reported for both groups (25). Another single-institution retrospective study comparing 52 hookwire with 57 methylene blue procedures reported a rate of $13 \%$ of dislodgements and one severe complication leading to patient's death (massive air embolism) in the hookwire group; moreover, an increased number of complications is reported for hookwire group, even though the difference is not statistically significant (overall complication rate $54 \%$ vs. $46 \%$; pneumothorax $38 \%$ vs. $25 \%$; perilesional haemorrhage $12 \%$ vs. $4 \%$ ). There were no significant differences in total procedure time, even in cases of dislodged wires; in these cases, surgical procedures were effective, since the lesion was identified through visualization of subpleural hematoma (26). In this study, hookwire was used in case of superficial nodules, while deeper nodules were marked with methylene blue (mean distance from pleura 9.2 vs. $14.7 \mathrm{~mm}, \mathrm{P}=0.01$ ), though Hwang reported no differences in distance from pleural surface between wire and coil groups $(9.1$ vs. $9.4 \mathrm{~mm}$, $\mathrm{P}=0.884$ ). Both the above reported studies comparing hookwire with other available CT-guided techniques showed similar localization effectiveness but more frequent and severe complications in the hookwire group, suggesting avoiding this marking method for deep lesions and being aware of potentially fatal complications. A recent metaanalysis including 46 studies and comparing the success and complication rates (both of preoperative localization and surgical identification of the markers) of hookwire, microcoil and lipiodol lung nodule localization methods for VATS procedures, reported optimal comparable results in preoperative targeting rates ( $>98 \%$ for all methods), but hookwire was associated with lower successful localization rate in operative field due to frequent dislodgement and higher complication rates (pneumothorax, haemorrhage and two cases of air embolism described only in the hookwire studies). Lipidol technique was associated with the best overall success rate $(99 \%)$ and microcoil localization yielded the lowest complication rates (27). Gonfiotti et al. published a prospective randomized study comparing hookwire and radio-guided localization techniques, reporting localization rates of $84 \%$ and $96 \%$ respectively; pneumothorax was observed in $24 \%$ patients of hookwire and in $4 \%$ of radio-guided group. Wire dislodgement and subsequent failure in identifying the lesion was reported in $12 \%$ of cases, mainly when the lesion was located in the 
posterior part of lower lobes. Authors concluded that, even if there were no statistical differences between the two groups, the radio-guided method appeared to be superior in detecting lung nodules (28). Main disadvantages of radioguided technique are the potential exposure to radioactive substances of personnel manipulating the surgical specimen (i.e., surgeons, nursing team, pathologists) and the need of a gamma-probe to intraoperatively identify the lesion. A systematic review by Zaman including 19 studies aimed to find the best way to locate a subcentimetre SPN among the available methods (intraoperative ultrasound, hook-wire, spiral-wire, fluoroscopic and radio-guided techniques): radio-guided surgical localization was reported as the preferable method, providing higher sensitivity, minimal operator-dependence and lower complication rates than other techniques (29).

In a HOR setting, the localization phase is conducted under general anaesthesia with the patient placed in a lateral decubitus, using a C-arm/O-arm cone-beam CT or MDCT. Various markers can be utilized, and VATS procedure is performed immediately after the localization (iVATS).

The main advantage using iVATS is the absence of waiting time between the localization procedure and the skin incision. On the other hand, it should be considered that HOR localization is associated with longer time under general anaesthesia, longer preparation time before surgery, global increased HOR occupation time, and overall higher costs if compared with conventional two-stage procedures. A recent study by Chao et al. reported a significantly higher time under general anaesthesia and HOR utilization time (mean time $227.4 v s .168 .7$ minutes) in patients undergoing intraoperative CT-guided nodule localization compared to preoperative CT-guided approach. Another concern about HOR procedure is radiation exposure: however, Chao et al. reported no significant differences between the two groups in localization procedural and radiation exposure (30).

Stanzi et al. reported encouraging results in terms of successful localization using both hookwires and coils, effectiveness of VATS procedures (only 1 conversion to thoracotomy out of 11 iVATS), overall procedural time, complication rates (no pneumothorax, no wire dislodgement) and radiation exposure using HOR and iVATS for non-palpable lung nodules (31).

In recent years, the increasing use of ENB has led to its application also as a localization method for non-palpable lung nodules: ENB-guided localization with fiducial markers or dyes can be performed during the same surgical time before VATS procedure, and allows marking of deep lung nodules without radiation exposure, with few reported complication rates (mainly pneumothorax): this method can also be applied for bilateral lesions, with a reduced risk of bilateral pneumothorax. Main disadvantages are the low cost/effectiveness and the increased time under general anaesthesia.

A retrospective propensity-matched analysis by Kuo et al. comparing ENB-guided patent vital blue injection with CTguided percutaneous localization reported no significant differences in the success rate, similar localization time (21.8-12.5 vs. 26.3-14.0 min) and surgical time, although CT-guided procedures were associated with higher complication rates, mainly with pneumothorax $(36.7 \%$ vs. $6.7 \%, \mathrm{P}=0.032$ ) (14). These results confirm the feasibility of this technique and the similar effectiveness if compared with CT-guided procedures.

In our experience, intraoperative identification of nonpalpable lung nodules still remains a surgical challenge: CT-guided localization methods with microcoils and dyes are routinely used at our institution to mark non-palpable subpleural GGO or subsolid lesions; recently, ENB-guided technique with radio-opaque fiducial marker placement was introduced and is currently being implemented with encouraging results. Unfortunately, at present time we do not have an HOR at our disposal, but we consider iVATS as one of the most promising and effective strategies not only to identify non-palpable lung nodules, but also to improve the accuracy of surgical procedures overall, especially in a future scenario of minimally-invasive surgery.

In conclusion, several localization techniques are available to successfully obtain an intraoperative identification of non-palpable lung nodules. Each technique has its advantages and disadvantages: the best approach should be selected considering the patient, the characteristic of the lesion (size, location, density) and the expertise of the medical staff (surgeons, interventional radiologists, specialists in nuclear medicine, bronchoscopists) in order to define an efficient and organized workflow to achieve the best results with minimal risks and discomfort for patients.

\section{Acknowledgments}

Funding: None.

\section{Footnote}

Provenance and Peer Review: This article was commissioned by the Guest Editors (Duilio Divisi and Roberto Crisci) for 
the series "Solitary Pulmonary Nodule" published in $A M E$ Surgical fournal. The article has undergone external peer review.

Reporting Checklist: The authors have completed the Narrative Review reporting checklist. Available at https:// asj.amegroups.com/article/view/10.21037/asj-21-17/rc

Peer Review File: Available at https://asj.amegroups.com/ article/view/10.21037/asj-21-17/prf

Conflicts of Interest: All authors have completed the ICMJE uniform disclosure form (available at https://asj.amegroups. com/article/view/10.21037/asj-21-17/coif). The series "Solitary Pulmonary Nodule" was commissioned by the editorial office without any funding or sponsorship. DT serves as an unpaid editorial board member of $A M E$ Surgical fournal from January 2021 to December 2022. The authors have no other conflicts of interest to declare.

Ethical Statement: The authors are accountable for all aspects of the work in ensuring that questions related to the accuracy or integrity of any part of the work are appropriately investigated and resolved.

Open Access Statement: This is an Open Access article distributed in accordance with the Creative Commons Attribution-NonCommercial-NoDerivs 4.0 International License (CC BY-NC-ND 4.0), which permits the noncommercial replication and distribution of the article with the strict proviso that no changes or edits are made and the original work is properly cited (including links to both the formal publication through the relevant DOI and the license). See: https://creativecommons.org/licenses/by-nc-nd/4.0/.

\section{References}

1. McWilliams A, Tammemagi MC, Mayo JR et al. Probability of cancer in pulmonary nodules detected on first screening CT. N Engl J Med 2013;369:910-9.

2. Park S, Park G, Lee SM, et al. Deep learning-based differentiation of invasive adenocarcinomas from preinvasive or minimally invasive lesions among pulmonary subsolid nodules [published online ahead of print, 2021 Feb 8]. Eur Radiol 2021. [Epub ahead of print]. doi: 10.1007/s00330-020-07620-z.

3. Tosi D, Mendogni P, Carrinola R, et al. CT-guided fineneedle aspiration biopsy of solitary pulmonary nodules under $15 \mathrm{~mm}$ in diameter: time for an afterthought? J Thorac Dis 2019;11:724-31.

4. Liu B, Gu C. Expert consensus workshop report: Guidelines for preoperative assisted localization of small pulmonary nodules. J Cancer Res Ther 2020;16:967-73.

5. Ichinose J, Kohno T, Fujimori S, et al. Efficacy and complications of computed tomography-guided hook wire localization. Ann Thorac Surg 2013;96:1203-8.

6. Hanauer M, Perentes JY, Krueger T, et al. Pre-operative localization of solitary pulmonary nodules with computed tomography-guided hook wire: report of 181 patients. J Cardiothorac Surg 2016;11:5.

7. Patella M, Bartolucci DA, Mongelli F, et al. Spiral wire localization of lung nodules: procedure effectiveness and oncological usefulness. J Thorac Dis 2019;11:5237-46.

8. McGuire AL, Vieira A, Grant K, et al. Computed tomography-guided platinum microcoil lung surgery: A cross-sectional study. J Thorac Cardiovasc Surg 2019;158:594-600.

9. Imperatori A, Fontana F, Dominioni L, et al. Videoassisted thoracoscopic resection of lung nodules localized with a hydrogel plug. Interact CardioVasc Thorac Surg 2019;29:137-43.

10. Vandoni RE, Cuttat JF, Wicky S, et al. CT-guided methylene-blue labelling before thoracoscopic resection of pulmonary nodules. Eur J Cardiothorac Surg 1998;14:265-70.

11. Park CH, Hur J, Lee SM et al. Lipiodol localization for Ground-glass opacity mInimal Surgery: Rationale and design of the LOGIS trial. Contemp Clin Trials 2015;43:194-9.

12. Grogan EL, Jones DR, Kozower BD, et al. Identification of small lung nodules: technique of radiotracer-guided thoracoscopic biopsy. Ann Thorac Surg 2008;85:S772-7.

13. Gobardhan PD, Djamin RS, Romme PJ, et al. The use of iodine seed (I-125) as a marker for the localization of lung nodules in minimal invasive pulmonary surgery. Eur J Surg Oncol 2013;39:945-50.

14. Kuo SW, Tseng YF, Dai KY, et al. Electromagnetic Naviglation Bronchoscopy Localization Versus Percutaneous CT-Guided Localization for Lung Resection via Video-Assisted Thoracoscopic Surgery: A PropensityMatched Study. J Clin Med 2019;8:379.

15. Gill RR, Zheng Y, Barlow JS, et al. Image-guided video assisted thoracoscopic surgery (iVATS) - phase I-II clinical trial. J Surg Oncol 2015;112:18-25.

16. Zhao ZR, Lau RWH, Yu PSY, et al. Devising the guidelines: the techniques of pulmonary nodule 
localization in uniportal video-assisted thoracic surgeryhybrid operating room in the future. J Thorac Dis 2019;11:S2073-S2078.

17. Melloni G, Venturino M, Mazza F, et al. Use of the hybrid room for thoracic surgery procedures: single-stage localization and removal of non-palpable nodules. Indian J Thorac Cardiovasc Surg 2021;37:70-7.

18. Kondo R, Yoshida K, Hamanaka K, et al. Intraoperative ultrasonographic localization of pulmonary ground-glass opacities. J Thorac Cardiovasc Surg 2009;138:837-42.

19. Khereba M, Ferraro P, Duranceau A, et al. Thoracoscopic localization of intraparenchymal pulmonary nodules using direct intracavitary thoracoscopic ultrasonography prevents conversion of VATS procedures to thoracotomy in selected patients. J Thorac Cardiovasc Surg 2012;144:1160-5.

20. Cuadrado DG, Grogan EL. Localization techniques for small lung nodules. Oper Tech Thorac Cardiovasc Surg 2014;19:179-98.

21. Lee NK, Park CM, Kang CH, et al CT-Guided Percutaneous Transthoracic Localization of Pulmonary Nodules Prior to Video-Assisted Thoracoscopic Surgery Using Barium Suspension. Korean J Radiol 2012;13:694-701

22. British Thoracic Society Pulmonary Nodule Guideline Development Group. Pulmonary Nodules I British Thoracic Society I Better lung health for all. Thorax 2015. Available online: https://www.brit-thoracic.org.uk/qualityimprovement/guidelines/pulmonary-nodules/

23. Sui X, Zhao H, Yang F, et al. Computed tomography guided microcoil localization for pulmonary small nodules and ground-glass opacity prior to thoracoscopic resection. J Thorac Dis 2015;7:1580-7.

doi: $10.21037 /$ asj-21-17

Cite this article as: Diotti C, Musso V, Mazzucco A, Tosi D. Preoperative localization of solitary pulmonary nodule: a narrative review. AME Surg J 2021;1:22.
24. McDermott S, Fintelmann FJ, Bierhals AJ, et al. Imageguided preoperative localization of pulmonary nodules for video-assisted and robotically assisted surgery. Radiographics 2019;39:1264-79.

25. Hwang S, Kim TG, Song YG. Comparison of hook wire versus coil localization for video-assisted thoracoscopic surgery. Thorac Cancer 2018;9:384-9.

26. Kleedehn M, Kim DH, Lee FT, et al. Preoperative pulmonary nodule localization: A comparison of methylene blue and hookwire techniques. AJR Am J Roentgenol 2016;207:1334-9.

27. Park CH, Han K, Hur J, et al. Comparative Effectiveness and Safety of Preoperative Lung Localization for Pulmonary Nodules: A Systematic Review and Metaanalysis. Chest 2017;151:316-28.

28. Gonfiotti A, Davini F, Vaggelli L, et al. Thoracoscopic localization techniques for patients with solitary pulmonary nodule: hookwire versus radio-guided surgery. Eur J Cardiothorac Surg 2007;32:843-47.

29. Zaman M, Bilal H, Woo CY, et al. In patients undergoing video-assisted thoracoscopic surgery excision, what is the best way to locate a subcentimetre solitary pulmonary nodule in order to achieve successful excision? Interact Cardiovasc Thorac Surg 2012;15:266-72.

30. Chao YK, Pan KT, Wen CT, et al. A comparison of efficacy and safety of preoperative versus intraoperative computed tomography-guided thoracoscopic lung resection. J Thorac Cardiovasc Surg 2018;156:1974-1983.e1.

31. Stanzi A, Mazza F, Lucio F, et al. Tailored intraoperative localization of non-palpablepulmonary lesions for thoracoscopic wedge resection using hybrid room technology. Clin Respir J 2018;12:1661-7. 